\title{
Motor unit number estimation versus compound muscle action potential in the evaluation of motor unit loss in amyotrophic lateral sclerosis
}

\author{
Arwa Khalid Ibrahem ${ }^{1}$, Akram Mohammed Al-Mahdawi and Farqad Bader Hamdan ${ }^{3^{*}}$ (D)
}

\begin{abstract}
Introduction: Motor neuron loss is the primary pathologic feature of amyotrophic lateral sclerosis (ALS). An estimate of the number of surviving motor units (MUs) represents a direct measure of the disease state in ALS. The objective of the study is to compare MU number estimation (MUNE) using the multipoint stimulation method (MPS) and compound muscle action potentials (CMAP) amplitude in patients with ALS.

Methods: Twenty-eight patients with ALS with a disease duration of 3-48 months were studied. Nerve conduction study of the median, ulnar, tibial, common peroneal, and sural nerves were done. Besides, electromyography (EMG) of cranio-bulbar, cervical, thoracic and lumbosacral muscles, and MPS-MUNE.

Results: MUNE is decreased in patients with ALS. MUNE was positively correlated with CMAP amplitude, medical research council (MRC) scale, and ALS functional rating scale (ALS-FRS). On the contrary, MUNE was negatively correlated with MUAP duration. Case detection by the MUNE methods was high as compared to that of CMAP amplitude.

Conclusions: MUNE is highly specific and more sensitive than CMAP amplitude in detecting the neurophysiologic abnormalities in patients with ALS. Case detection by MUNE is three times more than CMAP amplitudes. The rate of decline of motor units using the MPS-MUNE is more sensitive than the MRC score and ALSFRS-R when expressed as the percentage change from baseline.
\end{abstract}

Keywords: ALS, MPS-MUNE, CMAP amplitude, MRC scale, ALS-FRS

\section{Introduction}

Amyotrophic lateral sclerosis (ALS) is a neurodegenerative disorder. It is characterized by progressive degeneration and loss of motor neurons in the anterior horn cells of the spinal cord, motor nuclei of the brainstem, and the descending pathways within the corticospinal tracts [1].

In cases of rapid progression of the disease, the motor neuron or axon loss would be indicated as weakness and

\footnotetext{
* Correspondence: Farqadbhamdan@colmed-alnahrain.edu.iq; farqadbhamdan@yahoo.com

${ }^{3}$ Department of Physiology, College of Medicine, Al-Nahrain University, Neurophysiology Unit, Al-Immamain Al-Kadhimiyain Medical City, Baghdad, Iraq

Full list of author information is available at the end of the article
}

wasting. On the other hand, when the disorder is slowly progressive, loss of more than $50-80 \%$ of motor units (MUs) may occur with little or no clinically apparent weakness [2].

Verification of lower motor neuron abnormalities for definite ALS requires needle EMG in the muscles of at least three of four anatomic regions [3]. If abnormalities are not identified in any one area, but suspicion for an ALS remains high, the needle evaluation must expand. It may ultimately include an examination of muscles in all four regions [4], and this may increase patient discomfort and anxiety.

Using the principles of the revised El Escorial criteria, EMG evidence of acute denervation is limited to the 
presence of fibrillation or positive sharp waves. This constraint may not be as apparent in bulbar muscles or those muscles of normal bulk and strength which contribute to the fact that $22 \%$ of patients die from ALS without being assigned a level of certainty about the disease higher than the "clinically possible ALS" category [5].

Motor unit action potential (MUAP) recruitment analysis is a crucial aspect of the needle EMG examination where the majority of patients with ALS demonstrate recruitment abnormalities, even those examined during the early course of the disease [6].

Measurement of weakness is not a sufficient measure of the number of MUs because the surviving motor neurons keep pace with cell loss through collateral sprouting. The addition of new collaterals maintains muscle maximum compound muscle action potential (CMAP) amplitude [7], until late in the disease where collateral reinnervation is no longer able to provide full functional compensation creating MU instability [8]. Thus, normal CMAP amplitudes might mistakenly suggest that motor neuron loss has not occurred yet [9] and does not give the necessary information about the degree of MU loss [10]. Though, some studies reveal the CMAP amplitude is the only parameter with the advantage to demonstrate the progression of disease objectively in both ALS patients with poor and good prognosis [11].

Motor unit number estimate (MUNE) is a more reliable, physiologic technique than the CMAP amplitude in quantifying the numbers of MUs innervating an individual muscle and measure disease progression [4]. Therefore, it is an indirect measure of motor neuron loss, rather than a means of primary pathology. It can identify that the number of MUs may be well below average, in the presence of normal CMAP amplitudes [12].

The study aims to ameliorate the MUNE and CMAP amplitude in assessing the number of MU loss in patients with ALS.

\section{Methods}

A prospective two-center study was conducted at the departments of Neurology and Neurophysiology / Baghdad Teaching Hospital and Al-Imamain Al-Kadhimyain Medical City, Baghdad, Iraq for the period from Sept. 2017 till Feb. 2018. The Iraqi Board for Medical Specialization approved the study (Decision No. 860; Date 12/2/2018). Written informed consent was obtained from all individual participants included in the study.

Twenty-eight patients with ALS (either known cases or they were diagnosed during our workout) comprised 12 females and 16 males aged $55.2 \pm 12.312$ years (2469 years) with a duration of illness ranging from 3 to 48 months were studied. Patients with spinal muscular atrophy, progressive muscular atrophy, and Hirayama disease were excluded from the study.

Another 28 healthy subjects comprised of 13 females and 15 males aged $55.5 \pm 11.12$ years (range 24-65 years) serve as the control group.

Patients with ALS were subjected to a detailed history and clinical neurological examination adopting the El Escorial criteria [13]. Accordingly, nine patients fulfilled the criteria for definite ALS, 19 patients met the criteria for probable ALS, and none of them fulfilled the requirements for possible ALS. They were scored using the revised ALS Functional Rating scale (ALSFRS-R) which denotes 12 items (each of 5 scores from 0 to 4) [14]. Key muscles from the upper and lower extremities were evaluated using the Medical Research Council (MRC) scale against the examiner's resistance and grading the patient's strength on a 0 to 5 scale accordingly [15].

Neurophysiologic testing was done using Keypoint (Medtronic, Denmark) and Cadwell (USA) electromyography machines. The motor nerve conduction study of the median, ulnar, tibial, and fibular nerves and sensory nerve conduction study of the median, ulnar, and sural nerves were examined and the $\mathrm{F}$ wave latency of either lower limb following the standard methods [16]. The MRC scale and electromyographic activity were studied from the first dorsal interosseous (FDI) muscle. Twenty motor unit action potentials (MUAPs) were analyzed for duration and amplitude during minimal volitional effort. This is assessed by instructing the patient to make only a very gentle (low level) contraction of the muscle under investigation where single (first) MUP can be clearly differentiated on the monitor screen as it achieves a stable and fairly regular firing rate at $5-7 \mathrm{~Hz}$.

A multipoint stimulation (MPS-MUNE) method was used at the asymptomatic side or the less severely affected side (if any) by stimulating the ulnar nerve and recording from the abductor digiti minimi (ADM). The ulnar nerve was stimulated at three sites; $2 \mathrm{~cm}$ proximal to the wrist crease, $4 \mathrm{~cm}$ proximal to the first site, and 1 $\mathrm{cm}$ proximal to the ulnar groove at the elbow. At each side, we adjust the intensity at which we obtain the all or non-potential response which represent the single motor unit potential (SMUP). After recording one SMUP with a minimum amplitude of $\geq 25 \mu \mathrm{V}$, the stimulus intensity increased gradually by $4 \mathrm{~mA}$ for 10 times to have 10 SMUP at each site. The number of stimulations at each site should not be less than 4 and can be up to 10 . We did this procedure for each of the 3 sites of the nerve for each patient.

In severely affected patients with less than 10 SMUPs, as many as possible of SMUPs were recorded. MUNE can be calculated by dividing the supramaximal CMAP amplitude by the average amplitude of the $\sim 10$ SMUPs. 
The CMAP amplitude recorded from ADM muscle was measured from baseline to negative peak, and the CMAP with the highest amplitude is recorded.

\section{Statistical analysis}

Statistical analysis was performed using IBM-SPSS (statistical package for Social Sciences) version 25. Normal distribution of the data was assessed with the Kolmogorov-Smirnov test and variance homogeneity were evaluated with the Levene test. An independent $t$ test was used to analyze the difference between ALS patients and control groups concerning age, MUNE, CMAP amplitude, and SMUP. Chi-square test tests the association between gender with disease progression and age with disease progression.

Pearson's correlation examined the relationship between reduced FDI clinical function (as evaluated by the MRC scale and number of MUs calculated by MUNE); the relation between the MRC score and CMAP amplitude values, and the relation between disease duration, MUNE, MUAP parameters in FDI and SMUP.

ROC curve was used in the context of discrimination between MUNE and CMAP amplitude in detecting MU loss. According to this curve, the area under the curve (AUC), the sensitivity and specificity, and cut-off values were calculated.

The $p$ value of $\leq 0.05$ was considered statistically significant.

\section{Results}

The characteristics and neurophysiologic data of the ALS group (Table 1) illustrate that the CMAP amplitude was $4.68 \pm 3.14 \mathrm{mV}$, which is significantly $(p<0.001)$ lower than $23.4 \pm 8.6 \mathrm{mV}$ of the control group. Similarly, the MUNE of the patients with ALS was $107.89 \pm 53.7$, which is significantly reduced $(p<0.001)$ as compared to the $241.47 \pm 47.6$ of the control group.

On the contrary, the average SMUP area of patients with ALS was $4.8 \pm 2.9$, a significantly higher value $(p<0.001)$ than $0.1 \pm 0.04$ of the control group. Likewise, the SMUP amplitude of the patients with ALS was $85.7 \pm 67.1 \mu \mathrm{V}$ versus $49.05 \pm 8.5 \mu \mathrm{V}(p=0.003)$ of the control group (Table 2).

A positive correlation $(r=0.513, p=0.005)$ was demonstrated between the decreased number of MUs in ADM muscle (calculated by MUNE) and reduced FDI muscle clinical function (evaluated by the MRC scale). Similarly, the reduced CMAP amplitude was positively correlated $(r=0.520 ; p=0.007)$ with reduced FDI muscle function (evaluated by the MRC scale). Likewise, a positive correlation between CMAP amplitude and MUNE of ADM muscle $(r=0.621 ; p=0.001)$. Moreover, the ALS-FRS was positively correlated $(r=0.398$; $p=0.04$ ) with MUNE of ADM muscle (Fig. 1).
Table 1 The characteristics and clinical data of the patients

\begin{tabular}{ll}
\hline Character/parameter & \\
\hline Age (years) & $55.2 \pm 12.312$ (range 24-69) \\
Gender & \\
Females & $12(42.8 \%)$ \\
$\quad$ Males & $16(57.2 \%)$ \\
Disease duration (months) & $3-48$ \\
El Escorial criteria & \\
Definite & $9(32.1 \%)$ \\
Probable & $19(67.9 \%)$ \\
Possible & $0(0 \%)$ \\
Disease duration & \\
Slow & $23(82.1 \%)$ \\
Rapid & $5(17.9 \%)$ \\
Region of the disease onset & \\
Lower limbs & $14(50 \%)$ \\
Upper limbs & $12(42.9 \%)$ \\
Bulbar & $2(7.1 \%)$ \\
Associated disease & \\
None & \\
Bilateral carpal tunnel syndrome & $20(71.4 \%)$ \\
Liver transplantation & $3(10.7 \%)$ \\
Ulnar neuropathy & $1(3.6 \%)$ \\
Peripheral neuropathy & $1(3.6 \%)$ \\
Cervical spine surgery & $1(3.6 \%)$ \\
Lumber spine surgery & $1(3.6 \%)$ \\
ALS functional rating scale & $1(3.6 \%)$ \\
MRC score for FDI muscle & $36.79 \pm 8.556$ (range 13-46) \\
Mean MUAP duration of FDI muscle & 3.43 \\
Normal lower limb F wave & $21.1 \mathrm{~ms}$ \\
F repeater & $23(82.1 \%)$ \\
\hline & $5(17.9 \%)$ \\
\hline
\end{tabular}

FDI first dorsal interosseous, MUAP motor unit action potentials, data are presented in mean \pm SD or range or number (\%)

On the contrary, the reduced FDI muscle clinical function (evaluated by MRC scale) was negatively correlated $(r=-0.609 ; p=0.001)$ with MUAP duration of FDI. The ALS-FRS was negatively correlated $(r=-0.552$; $0.002)$ with MUAP duration of FDI muscle. The MUAP duration was also negatively correlated $(r=-0.540$; $p=0.003)$ with MUNE of ADM muscle.

The ALS disease duration was positively correlated $(r=0.852 ; p=0.011)$ with the MUAP duration of the FDI muscle (Fig. 2).

Table 2 The differences in the MPS MUNE, CMAP amplitude, and average SMUP area between ALS patients and control group (independent $t$ test)

\begin{tabular}{llll}
\hline Variable & $\begin{array}{l}\text { ALS patients } \\
\boldsymbol{n}=\mathbf{2 8}\end{array}$ & $\begin{array}{l}\text { Control group } \\
\boldsymbol{n}=\mathbf{2 8}\end{array}$ & $\boldsymbol{p}$ value \\
\hline CMAP amplitude $(\mathrm{mV})$ & $4.68 \pm 3.14$ & $23.4 \pm 8.6$ & $<0.001$ \\
MPS MUNE & $107.89 \pm 53.7$ & $243.47 \pm 47.6$ & $<0.001$ \\
Average SMUP area \% & $4.8 \pm 2.9$ & $0.1 \pm 0.04$ & $<0.001$ \\
SMUP amplitude $(\mu \mathrm{V})$ & $85.7 \pm 67.1$ & $49.05 \pm 8.5$ & 0.003
\end{tabular}

ALS amyotrophic lateral sclerosis, CMAP compound muscle action potential, MPS MUNE multipoint stimulation motor unit number estimation, SMUP single motor unit potential. (Ulnar nerve stimulation and recording from the ADM muscle) 

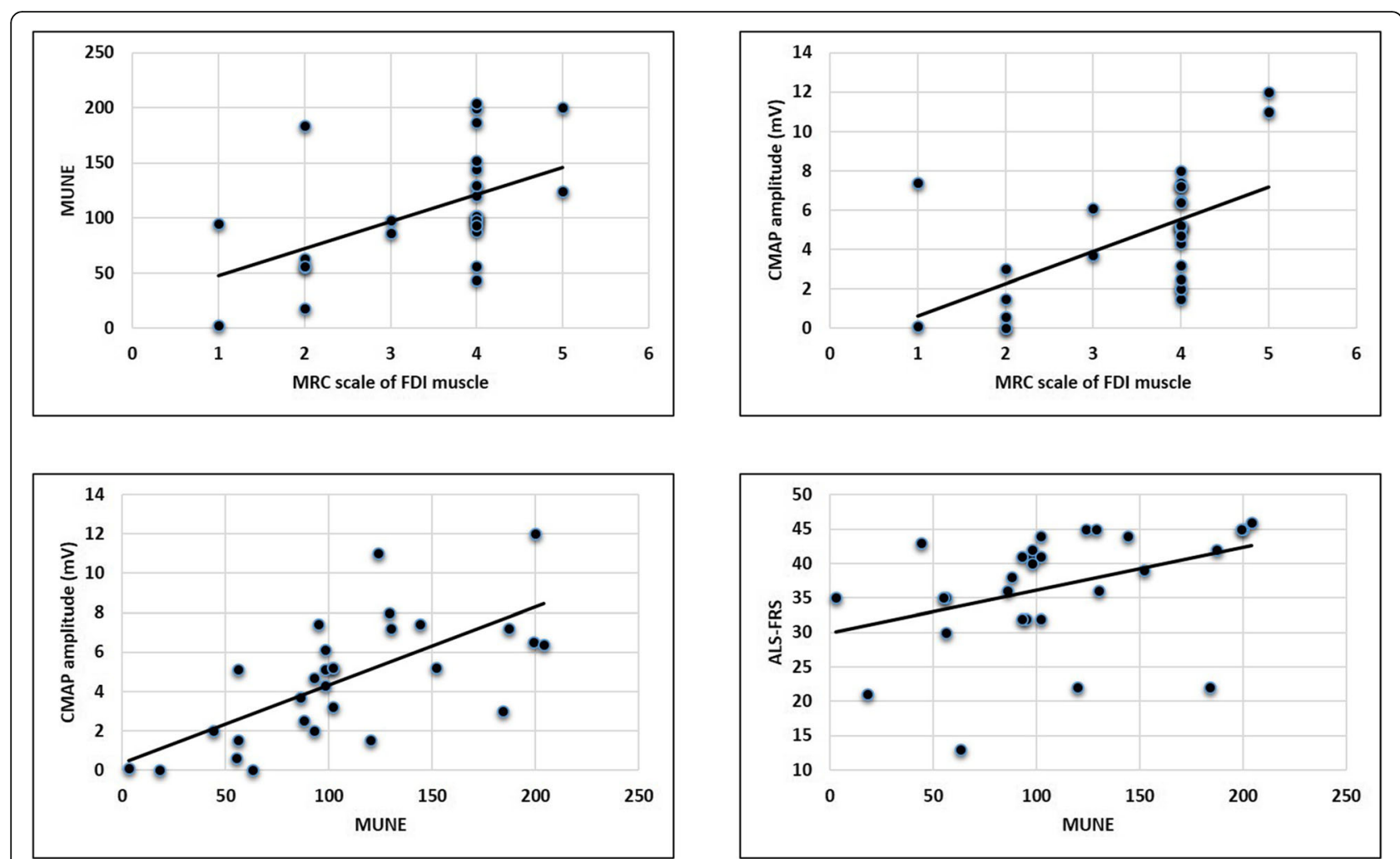

(lower left); MUNE and the ALS-FRS (bottom right)

No significant correlation was demonstrated between the reduced function of FDI (evaluated by MRC scale) and SMUP $(r=-0.250 ; p=0.199)$ and between the CMAP amplitude and MUAP duration of FDI muscle $(r=-0.193 ; p=0.344)$. Furthermore, no correlation was noticed between the gender and the ALS disease progression, MUNE of ADM muscle and each of the onset region, disease duration, disease progression, and MUAP duration $(p>0.05)$.

Case detection using the MUNE versus CMAP amplitude (Table 3) demonstrate that the MUNE has high sensitivity $80.8 \%$ and $95 \%$ specificity as compared to the CMAP amplitude (30.8\% sensitivity and $95 \%$ specificity).

\section{Discussion}

MUNE decrement in patients with ALS reflects the dropout of MUs in the disease process. This finding was also noticed by other researchers elsewhere [17, 18]. Not surprisingly, the CMAP amplitude is significantly reduced in patients with ALS reflecting a continuous axonal loss. Moreover, the present study demonstrates a significant positive correlation between the CMAP amplitude and MUNE values. This relationship was expected because CMAP amplitude as a measure is used in the calculation of MUNE values. This finding was in harmony with the observation of other researchers [7].
In our study, CMAP amplitude was also significantly correlated with the MRC scale. A finding was also observed by Onesti et al. [11]. In ALS, the recruitment frequency is increased because fewer anterior horn cells are available to be activated as effort increases. When recruitment is reduced (as low MRC scale) from a loss of anterior horn cells, the number of MUs firing at any given firing rate is reduced $[4,19]$. On the other hand, ALS-FRS was positively correlated with MUNE of ADM muscle; this could be ascribed to the sufficient reinnervation despite clinical disease progression. Furthermore, changes in MUNE values can be seen before clinically detectable decreased force and atrophy [20].

On the contrary to the results of our study, Kristensen et al. [21] demonstrate no significant correlation between ALSFRS-R and either of MUNE, MUAP duration, or CMAP amplitude. Similarly, Jacobson et al. [7, 22] found no relationship between ALS-FRS and all MUNE methods except for MScan, yet, in their study, ALS-FRS reflects the global function of all muscles tested while it demonstrates the role of only one muscle in our study.

MUNE was positively correlated with the MRC score and the clinical dysfunction measured by the ALSFRS-R scale. This finding was in agreement with other researchers, although using MUNIX instead of MUNE as a measure $[23,24]$. MUNE is calculated by dividing 

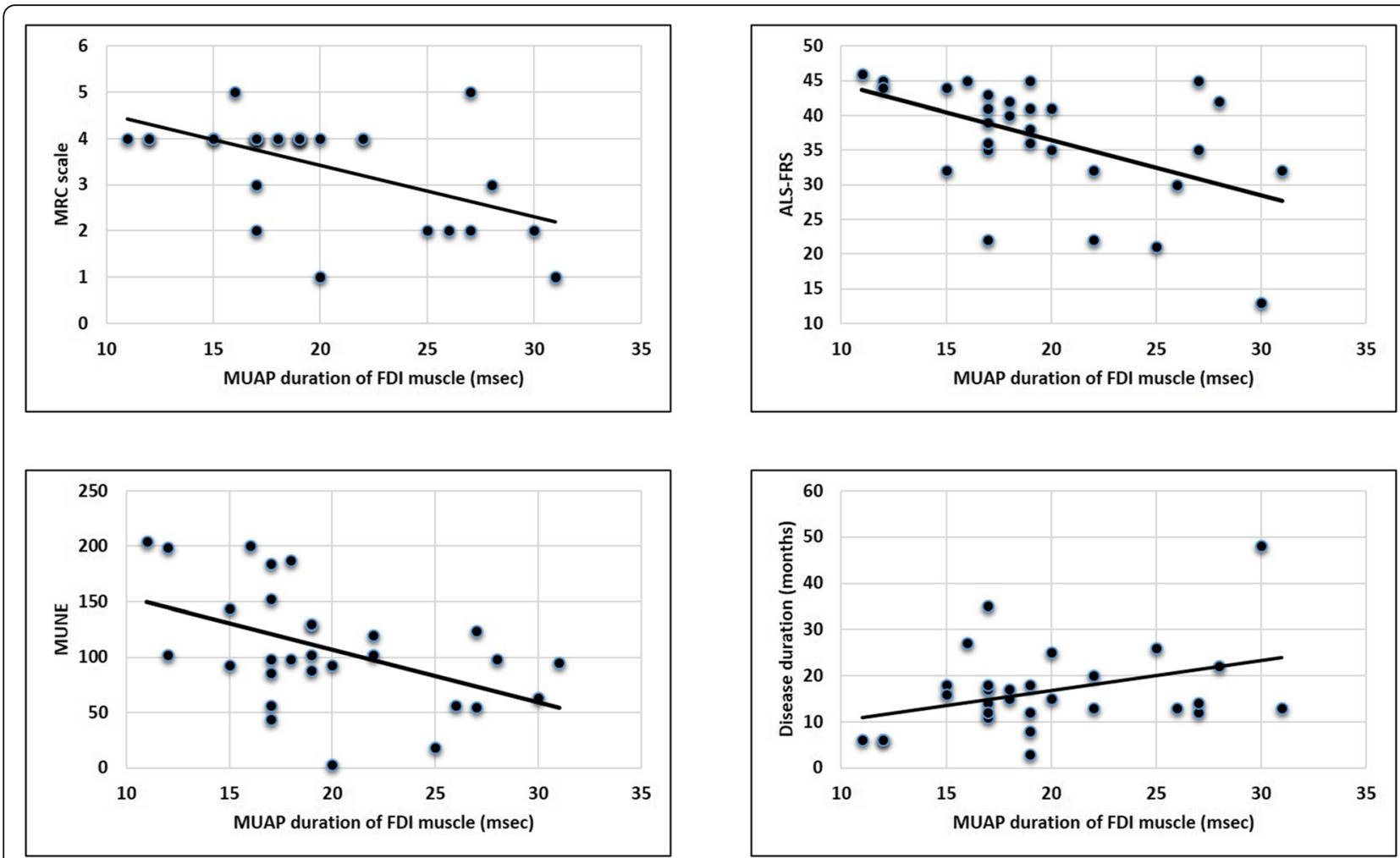

Fig. 2 Correlation between the MRC scale and the MUAP duration (upper left); the ALSFRS and the MUAP duration (top right); MUAP duration and MUNE (bottom left); the MUAP duration and disease duration

the maximal CMAP amplitude by the mean surface SMUP [25]. In contrast, MUNIX is derived from a mathematical model based on CMAP amplitude and electromyographic surface interference patterns [26]. Yet, MUNE and MUNIX scores are inter-correlated in patients with ALS [27].

No correlation was noticed between the MUNE and disease progression which contradicts the results of Ahn et al. [28] and Jacobsen et al. [29] because the latter groups used the statistical MUNE and revealed that the MUNE ratio of SW-MUNE was coincident with the clinical rapidity of disease progression by ALS-FRS.

It was well known that ALS-FRS evaluated the disease progression; unfortunately, our patients were not followed neurophysiologically because of the short timetable plus some of the patients skipped as they were referred from other centers. It worth saying that following up the disease progression should be done using MUNE in its different methods because it is more sensitive than ALS-FRS as proved by several studies [20,30].

MUNE of ADM muscle was negatively correlated with MUAP duration of FDI muscle in our study. Jacobson et al. [22] were the first to study the relation of MUNE and MUAP duration in the same muscle, which is the FDI. In their earlier trials, no correlation was observed between MUAP parameters in the FDI muscle and adapted MPS MUNE in ADM muscle, used more than one MUNE method, and found that none of the MUNE parameters correlated to MUAP duration or amplitude. The limitation of that study was attributed to their use of ADM muscle in MUNE and FDI muscle for MUAP analyses. Although the ulnar nerve innervates both muscles, the affection of the ADM and FDI may be different because of the split hand phenomenon. The latter could

Table 3 Diagnostic sensitivity and specificity of MPS MUNE and CMAP amplitude in ALS patients

\begin{tabular}{llllllll}
\hline Factor & Cut-off & AUC & Sensitivity & Specificity & Upper bound & Lower bound & $\boldsymbol{p}$ value \\
\hline MUNE & 180.5 & 0.965 & $80.8 \%$ & $95 \%$ & 0.922 & 1.000 & $0.000^{*}$ \\
CMAP amplitude & 3.4 & 0.611 & $30.8 \%$ & $95 \%$ & 0.447 & 0.774 & 0.203 \\
\hline
\end{tabular}

AUC area under the curve, MPS MUNE multipoint stimulation motor unit number estimation, CMAP compound muscle action potentials. (UInar nerve stimulation and recording from the ADM muscle) 
explain why changes in MUAP parameters that occur in the course of denervation and reinnervation does not reflect the exact degree of MU damage.

The negative correlation between MUAP duration of the FDI muscle and MRC score is supported by the findings of Gawel et al. [23] who demonstrated no correlation between MUAP parameters including amplitude, duration, and muscle function assessment by the MRC scale. Likewise, MUAP duration of the FDI muscle was negatively correlated with ALS-FRS, which is in harmony with the results of Jacobson et al. [22]. MUAP with increased duration would reflect chronic denervation and reinnervation, which means some of the MUs have died as revealed by a reduced ALS-FRS.

Case detection by the MUNE methods was high as compared to that of CMAP amplitude reflected as high sensitivity and specificity. Jacobsen et al [29] also evaluate the MUNE method and MScan as a measure of disease progression in ALS compared with CMAP amplitude and found MScan registered the largest decline (8.7\% per month), compared with MPS (3.4\%), and CMAP amplitude (2.0\%). This low specificity and sensitivity support the notable variation in normal values for the CMAP amplitude and limited reproducibility with retesting [12] as compared to the MUNE method.

\section{Conclusion}

MUNE is correlated well with CMAP amplitude, MUAP duration, and functional scoring in patients with ALS. MUNE methods are found to be highly specific and more sensitive than CMAP amplitude in evaluating MU loss in patients with ALS, case detection by MUNE is three times more than CMAP amplitudes. Finally, the rate of decline of MUs using the multipoint incremental MUNE is more sensitive than that of MRC score and ALSFRS-R, when expressed as the percentage change from baseline.

The small number of patients is a limitation in this study but is comparable with other similar studies and did not allow us to do analyses for subgroups of patients with differing disease onset (bulbar vs limb).

\section{Abbreviations}

ADM: Abductor digiti minimi; ALS: Amyotrophic lateral sclerosis.; ALSFRS: ALS functional rating scale; CMAP: Compound muscle action potential.; EMG: Electromyography; FDI: First dorsal interosseous; MPS: Multipoint stimulation.; MRC: Medical research council; MU: Motor units; MUNE: Motor unit number estimation; SMUP: Single motor unit potential

\section{Acknowledgements}

We thank Mr. M. Maan from the College of Medicine, Baghdad University, for helping us in the statistical analysis.

\section{Settings}

Neurophysiology Unit, Al-Imamain Al-Kadhimyian Medical City, Baghdad, Iraq.

\section{Authors' contributions}

All the authors have directly participated in the preparation of this manuscript and have approved the final version submitted. AA examined and referred the patients. AKI collected the cases and drafted the manuscript. FBH and AA conceived the study and participated in its design, interpretation, and supported manuscript drafting. All the authors have read and approved the final manuscript.

\section{Funding}

Self-funding

\section{Availability of data and materials}

All data generated or analyzed during this study are included in this published article. The datasets used and/or analyzed during the current study are available from the corresponding author on reasonable request.

\section{Declarations}

\section{Ethics approval and consent to participate}

The study design was approved by the Iraqi Board for Medical Specialization (Decision No. 860; date 12 February 2018). An informed written consent was obtained from all participants.

\section{Consent for publication}

Not applicable

\section{Competing interests}

The authors declare that they have no competing interest.

\section{Author details}

${ }^{1}$ Neurophysiology Unit, Neurosurgery Teaching Hospital, Al-Risafa Health Directorate, Ministry of Health, Baghdad, Iraq. ${ }^{2}$ Scientific Council of Neuromedicine, Iraqi Board for Medical Specialization, Baghdad, Iraq. ${ }^{3}$ Department of Physiology, College of Medicine, Al-Nahrain University, Neurophysiology Unit, Al-Immamain Al-Kadhimiyain Medical City, Baghdad, Iraq.

Received: 26 October 2020 Accepted: 22 March 2021

Published online: 31 March 2021

\section{References}

1. Saberi S, Stauffer JE, Schulte DJ, Ravits J. Neuropathology of amyotrophic lateral sclerosis and its variants. Neurol Clin. 2015;33(4):855-76. https://doi. org/10.1016/j.ncl.2015.07.012.

2. Maathuis EM, Drenthen J, van Doorn PA, Visser GH, Blok JH. The CMAP scan as a tool to monitor disease progression in ALS and PMA. Amyotroph Lateral Scler Frontotemporal Degener. 2013;14(3):217-23. https://doi.org/1 0.3109/21678421.2012.732079.

3. de Carvalho M, Dengler R, Eisen A, England JD, Kaji R, Kimura J, et al. Electrodiagnostic criteria for diagnosis of ALS. Clin Neurophysiol. 2008; 119(3):497-503. https://doi.org/10.1016/j.clinph.2007.09.143.

4. Joyce NC, Carter GT. Electrodiagnosis in amyotrophic lateral sclerosis. PM R. 2013;5(5 Suppl):S89-95. https://doi.org/10.1016/j.pmrj.2013.03.020.

5. Traynor BJ, Codd MB, Corr B, Forde C, Frost E, Hardiman OM. Clinical features of amyotrophic lateral sclerosis according to the El Escorial criteria and Airlie house diagnostic criteria. Arch Neurol. 2000;57(8):1171-6. https:// doi.org/10.1001/archneur.57.8.1171.

6. Inghilleri M, lacovelli E. Clinical neurophysiology in ALS. Arch Ital Biol. 2011; 149(1):57-63. https://doi.org/10.4449/aib.v149i1.1264.

7. Jacobsen $A B$, Bostock $H$, Fuglsang-Frederiksen A, Duez L, Beniczky S, Møller AT, et al. Reproducibility, and sensitivity to motor unit loss in amyotrophic lateral sclerosis, of a novel MUNE method: MScanFit MUNE. Clin Neurophysiol. 2017;128(7):1380-8. https://doi.org/10.1016/j. clinph.2017.03.045.

8. Iwai Y, Shibuya K, Misawa S, Sekiguchi Y, Watanabe K, Amino H, et al. Axonal dysfunction precedes motor neuronal death in amyotrophic lateral sclerosis. PLoS One. 2016;11(7):e0158596. https://doi.org/10.1371/journal. pone.0158596.

9. Shefner JM. Motor unit number estimation in human neurological diseases and animal models. Clin Neurophysiol. 2001;112(6):955-64. https://doi.org/1 0.1016/S1388-2457(01)00520-X. 
10. Jacobsen AB, Bostock H, Tankisi H. CMAP scan MUNE (MScan) - a novel motor unit number estimation (MUNE) method. J Vis Exp. 2018 ; 136:e56805.

11. Onesti E, Gori MC, Ceccanti M, Tartaglia G, Petrucci A, Frasca V, et al. The compound muscle action potential as neurophysiological marker for amyotrophic lateral sclerosis. EC Neurol. 2016;3:509-19.

12. Henderson RD, McCombe PA. Assessment of motor units in neuromuscular disease. Neurotherapeutics. 2017;14(1):69-77. https://doi.org/10.1007/ s13311-016-0473-z.

13. Ludolph A, Drory V, Hardiman O, Nakano I, Ravits J, Robberecht W, et al. A revision of the El Escorial criteria - 2015. Amyotroph Lateral Scler Frontotemporal Degener. 2015;16(5-6):291-2.

14. Swash M. New ideas on the ALS functional rating scale. J Neurol Neurosurg Psychiatry. 2017;88(5):371-2. https://doi.org/10.1136/jnnp-2 016-315116.

15. Ciesla N, Dinglas V, Fan E, Kho M, Kuramoto J, Needham D. Manual muscle testing: a method of measuring extremity muscle strength applied to critically ill patients. J Vis Exp. 2011; (50): pii 3632.

16. Kimura J. Electrodiagnosis in diseases of nerve and muscle. In: Principles and Practice. 4th (ed.) ed. New York: Oxford University Press; 2013.

17. Jagtap SA, Kuruvilla A, Govind P, Nair MD, Sarada C, Varma RP. Multipoint incremental motor unit number estimation versus amyotrophic lateral sclerosis functional rating scale and the medical research council sum score as an outcome measure in amyotrophic lateral sclerosis. Ann Indian Acad Neurol. 2014;17(3):336-9. https://doi. org/10.4103/0972-2327.138522.

18. de Carvalho M, Barkhaus PE, Nandedkar SD, Swash M. Motor unit number estimation (MUNE): Where are we now? Clin Neurophysiol. 2018;129(8): 1507-16. https://doi.org/10.1016/j.clinph.2018.04.748.

19. Gaweł M. Electrodiagnostics: MUNE and MUNIX as methods of estimating the number of motor units - biomarkers in lower motor neurone disease. Neurol Neurochir Pol. 2019;53(4):251-7. https://doi. org/10.5603/PJNNS.a2019.0026.

20. Neuwirth C, Barkhaus PE, Burkhardt C, Castro J, Czell D, de Carvalho M, et al. Motor Unit Number Index (MUNIX) detects motor neuron loss in presymptomatic muscles in Amyotrophic Lateral Sclerosis. Clin Neurophysiol. 2017;128(3):495-500

21. Kristensen RS, Jacobsen AB, Fuglsang-Frederiksen A. Motor unit number estimation methods compared to quantitative motor unit potential analysis in amyotrophic lateral sclerosis. Clin Neurophysiol. 2017;128(9):e188. https:// doi.org/10.1016/j.clinph.2017.07.036.

22. Jacobsen AB, Kristensen RS, Witt A, Kristensen AG, Duez L, Beniczky S, et al. The utility of motor unit number estimation method versus quantitative motor unit potential analysis in diagnosis of ALS. Clin Neurophysiol. 2018;129(3):646-53. https://doi.org/10.1016/j.clinph.2018. 01.002 .

23. Gaweł M, Kuzma-Kozakiewicz M. Does the MUNIX method reflect clinical dysfunction in amyotrophic lateral sclerosis: a practical experience. Medicine (Baltimore). 2016;95(19):e3647. https://doi.org/10.1 097/MD.0000000000003647.

24. Wirth AM, Khomenko A, Baldaranov D, Kobor I, Hsam O, Grimm T, et al. Combinatory biomarker use of cortical thickness, MUNIX, and ALSFRS-R at baseline and in longitudinal courses of individual patients with amyotrophic lateral sclerosis. Front Neurol. 2018;9:614. https://doi.org/1 0.3389/fneur.2018.00614

25. Gooch CL, Doherty TJ, Chan KM, Bromberg MB, Lewis RA, Stashuk DW, et al. Motor unit number estimation: a technology and literature review. Muscle Nerve. 2014;50(6):884-93. https://doi.org/10.1002/mus.24442.

26. Grimaldi S, Duprat L, Grapperon AM, Verschueren A, Delmont E, Attarian S. Global motor unit number index sum score for assessing the loss of lower motor neurons in amyotrophic lateral sclerosis. Muscle Nerve. 2017;56(2): 202-6. https://doi.org/10.1002/mus.25595

27. Stein F, Kobor I, Bogdahn U, Schulte-Mattler WJ. Toward the validation of a new method (MUNIX) for motor unit number assessment. J Electromyogr Kinesiol. 2016;27:73-7. https://doi.org/10.1 016/j.jelekin.2016.02.001.

28. Ahn SW, Kim SH, Oh DH, Kim SM, Park KS, Hong YH, et al. Motor unit number estimation in evaluating disease progression in patients with amyotrophic lateral sclerosis. J Korean Med Sci. 2010;25(9):1359-63. https:// doi.org/10.3346/jkms.2010.25.9.1359.
29. Jacobsen $A B$, Bostock $H$, Tankisi $H$. Following disease progression in motor neuron disorders with 3 motor unit number estimation methods. Muscle Nerve. 2019;59(1):82-7. https://doi.org/10.1002/mus.26304.

30. Boekestein WA, Schelhaas HJ, van Putten MJ, Stegeman DF, Zwarts MJ, Van Dijk JP. Motor unit number index (MUNIX) versus motor unit number estimation (MUNE): a direct comparison in a longitudinal study of ALS patients. Clin Neurophysiol. 2012;123(8):1644-9.

\section{Publisher's Note}

Springer Nature remains neutral with regard to jurisdictional claims in published maps and institutional affiliations.

\section{Submit your manuscript to a SpringerOpen ${ }^{\circ}$ journal and benefit from:}

- Convenient online submission

- Rigorous peer review

- Open access: articles freely available online

High visibility within the field

- Retaining the copyright to your article

Submit your next manuscript at $\boldsymbol{\sim}$ springeropen.com 